\title{
Study on Shell Sort of Logistics Unified Information System based on Internet of Things
}

\author{
Xingzhi Lin \\ Guangxi Economic Management Cadre College \\ Nanning,Guangxi,China \\ E-Mail: lxz4562509@139.com
}

\begin{abstract}
Through the analyze on the technology, information data processing of logistics unified information system, the optimization Algorithm of Shell Sort in logistics unified information system data processing is proposed. Things-Based Logistics Unified Information System is an integrated system which combine with integration of RFID (Radio Frequency Identification, RFID), infrared sensors, global positioning systems, laser scanners and other telecommunications integrated fusion machine (Computer Telecommunication Integration, CTI) technology. The improved Shell sort algorithm is used in system data processing, so that multiple devices, multi-technology, multi-interface, and distributed widely disorder high-speed logistics systems to available for the heterogeneous applications and fast data processing. It improves multiple-concurrent data processing. Based on JAVA programming of Shell sort, to build and test a system algorithm, which support the system with a high-speed interactive logistics business processing capabilities. The design and implementation feasibility of Shell sort algorithm is proved in Internet of Things-Based Logistics Unified Information System through the output of test results.
\end{abstract}

Keywords- Internet Of Things;Logistics; Unified Information System;Shell Sort

\section{INTRODUCTION}

Logistics Unified Information System is a system application and function extension of Internet of Things, which adopts the technical characters of thing-to-thing of internet, via RFID(Radio Frequency Identification), infrared sensor, GPS, laser scanner as well as combination of computer telecommunication integration and other information technologies and transmission equipments, based on the agreed protocol, realizes the intelligent identification, positioning, tracking, monitoring and management of each link of logistics flow[1]. In the Logistics Unified Information System business flow, there's huge amount of unsorted data flow, application of Shell Sort algorithm provides possibility of optimization process to multiple concurrent data chains of the Logistics Unified Information System, meanwhile, it provides bigger development space for IOT information process flow. Shell Sort belongs to category of Insertion Sort, which adopts function of Step Serial to simplify and resolve time complexity and space complexity. Selecting proper Step Serial in the Logistics Unified Information System based on IOT technology, enables relatively less comparison and transfer during the sorting process, in order to adapt to unified information data flow of distributed aggregated data chain, and with the quick variation of the serial record number, the time complexity of Shell algorithm could be optimized[2].

The data process of Logistics Unified Information System based on IOT is a type of creative mode combining CTI and Internet technology, the IOT technology is applied to all sorts of application tools and information services provided by mobile phone, PDA, radio collector, laptop and Internet, and forms integrated unified information logistics business flow process service chain[3]. The collection process of the unified information has lots of irregularities and random, the system devices represented by GPRS and other mobile devices is equipped with random features in real time, it needs a sort algorithm like Shell to realize intelligent coordinate process and technical support to the system, in order to handle the characters of comprehensive distribution and high speed access of the IOT technology.

\section{INTRODUCTION OF SHELL SORT FOR LOGISTICS UNIFIED INFORMATION SYSTEM}

Logistics Unified System based on IOT could be divided into four layers in terms of application, as shown in figure 1 shows:

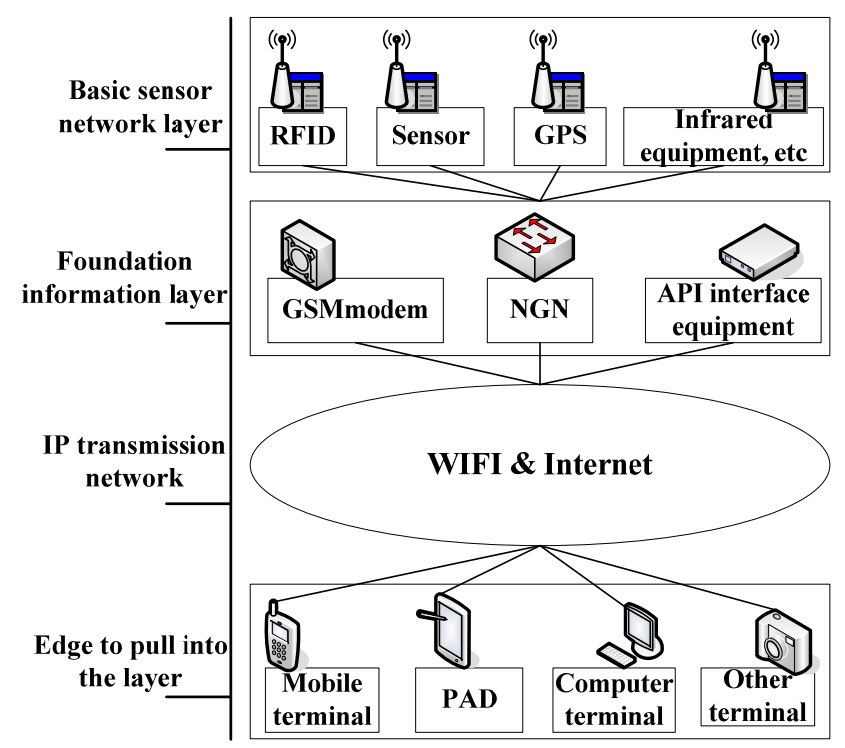

Figure 1. Based on the content of the networking unified logistics information system structure 
- $\quad$ First it's basic transmission network of IOT, mainly via FRID, sensor and GPS, it realizes wide distributed collection and thing-to-thing interconnection of logistics unified information[4].

- $\quad$ Secondly is the basic information network of Unified Information System, supported by CTI and internet technology, it adopts GSM modem, NGN and other new devices, could based on API interface develop exchangeable voice, video, information and other special value added services of logistics unified information.

- Thirdly it's IP transmission network, including wire and wireless networks, which via internet, telecom network, broadcasting network or next generation internet realizes transmission and calculation of distributed data.

- Fourthly it's edge inclusion layer, this is the bottom layer applied to user terminal, the main devices are IOT, telecom network, internet and all types of input and output control terminals.

From the four layers of IOT application, it can be learnt that the transmission and application difference between transmission network and application network is not big, the key lies on the information identification of realizing Things[5]. In the logistics supply chain of Unified Information System, RFID tab stores the logistics goods information and converge to Unified Information System via all types of communication networks, to realize data process as well as information exchange and sharing during the logistics flow[6]. During the information exchange of these four layers, Shell Sort algorithm plays a role of data process guidance and media, this performs group optimization and process for step serial of each element. Shell sort realize as shown in figure 2 shows:

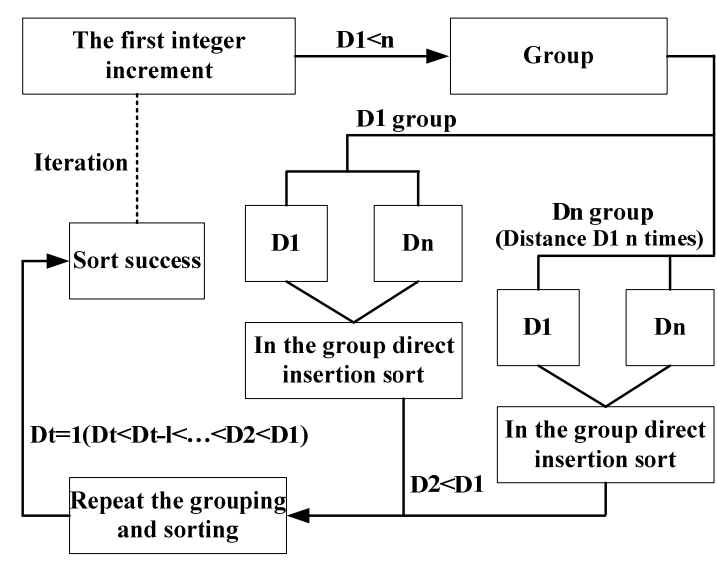

Figure 2. Shell sort realize

Shell Sort is an operation to use a serial of record, based certain element value or key word, to put into sequence by progressive increase or decrease. In IOT Unified Information System, the Shell Sort algorithm steps are: 1 . Select the first integer increment: $\mathrm{D} 1<\mathrm{n} ; 2$. Group: divide into D1 groups, the records with distance of $\mathrm{N}$ times of $\mathrm{D} 1$ are in the same group; 3. Insert into sequence within the group; 4 . Select the second integer increment: D2<D1, 5. Repeat aforementioned groups and sequences, and meet increment conditions: $\mathrm{Dt}=1(\mathrm{Dt}<\mathrm{Dt}-\mathrm{l}<\ldots<\mathrm{D} 2<\mathrm{D} 1)$, realize all records being able to directly insert to sequence within the same group.

In order to realize Shell Sort inserting sequence with different increment groups each time in Logistics Unified Information System based on IOT, when inserting sequence for the last time, all the records should be sequential, only in this way the unsorted IOT information collection can exist in sorted unified information process, to prevent overflow due to widely and extensively existing huge amount of unsorted unified information data[7]. Therefore, all the increments in Shell Sort algorithm should be bigger than 1, and jumpily move inverted sequential records, in order to speed up sorting process.

\section{DESIGN CONCEPT AND REALIZATION}

\section{A. Design Concept.}

Based on the characters of IOT technology and unified information process flow, it adopts improved Shell Sort algorithm, the design concept is:

- Sub-serial grouping: divide the whole record serial waiting to sort into several sub-serials;

- Directly insert into sequence: perform directly insertion into the sequence for each sub-serial respectively;

- Basically sequential: realize basically sequential record in sub-serials;

- All-recorded sequence: Implement a direct insertion sort for overall records.

In the case of positive sequence for the record serial, the time complexity could be lifted to higher sequence.

Assuming sequence pending for sorting is basically sequential, which is:

L.R[i].key $<\operatorname{Max}\{L . R[j]$. key $\}$

$1 \leqslant \mathrm{j}<\mathrm{i}$, with less recorded sequences, the efficiency of direct insertion sort could be significantly enhanced; Otherwise, when the value is small the efficiency could be relatively high as well.

Composition of sub-serial of Shell Sort: it's not simply to divide each data piecemeal but put records with interval of certain increment into a sub-serial. Compare the key word recorded in inserted sequence of previous one or two rounds with the last key word recorded in the same sequence, realize record of small key word jumpily being moved forward, when implementing the insertion sort with last round of increment of 1 , the serial has been basically sequential, only by recording small amount of comparison and moving, the sorting could be completed.

\section{void ShellInsert (SqList \&L, int Dk)}

For sequence list $\mathrm{L}$ implement a round of Shell insertion sort, comparing with the first round of direct insertion sort, the algorithm could be modified as following:

- The integer increment of the before and after recorded pointer is Dk instead of 1 ; 
- $\mathrm{R}[0]$ is only the temporary storage unit of the serial;

- When $\mathrm{j}<=0$, find the insertion pointer for the serial elements.

$$
\text { for }(i=D k+1 ; i<=\text { L.Length } ;++i)
$$

The record with small key word is jumpily moved forward, when the increment is 1 , the serial has been basically sequential, it needs to insert L.R[i] into serial increment sub-list:

\section{if $L T(L . R[i] . k e y, \quad L . R[i-D k] . k e y)$}

For those key words undefined temporarily, they could not be directly inserted into the increment list, but temporarily stored in L.R[0]:

$$
\begin{aligned}
& L . R[0]=L . R[i] ; \\
& \text { for }(j=i-D k ; \\
& \quad j>0 \& \& L T(L . R[0] . k e y, L . R[j] . k e y) ; j-=D k)
\end{aligned}
$$

The key words recorded in insertion sort are compared with the key words of the last record in the same sub-serial, and moved afterward after recording; the insertion locations are located and then insert:

$L \cdot R[j+D k]=L \cdot R[j]$

$L . R[j+D k]=L . R[0]$;

According to the increment serial Dlta[0..t-1], implement Shell Sort on sequence list L:

$$
\text { void ShellSort (Sqlist \&L, int Dlta[], int t) }
$$$$
\text { for }(k=0 ; k<t ;++t)
$$

A round of insertion sort with increment of Dlta[k]:

ShellInsert(L,Dlta[k]);

Increment serial could have many methods for selection, but it needs to ensure in the increment serial there's no common factor except 1, and the final value must be 1 .

\section{B. Realization of Shell Sort.}

In the Unified Information System based on IOT, the process of logistics information data is mainly realized through programming, intelligent read on all types of business data is realized via intelligent module of unified information. Shell Sort adopts JAVA for program realization, part of the codes are as following:

\section{public class Test \{}

In the system, first of all select increment for a group of integer. Predefine data groups, the integer increments are as following:

public static int [ ] $a=\{7,23,6,13,4,9,17,21,0,31\}$;

public static void main(String args [ ]) \{

Among elements of each serial, it needs to design cycle counting variable (i), data index variable etc; result U before serial output:

int $i$;

int Index = a.Length;

System.out.print("U: ");

for $(i=0 ; i$ \&lt; Index $-1 ; i++)$

System.out.printf("\%3s ", a);

System.out.println("'");
In the serial, it needs to select sequence based on each key word, in order to arrange for the sequence of the next step:

ShellSort(Index - 1);

After sorting, it needs to display the result; $\mathrm{M}$ represents the displayed result after sorting:

System.out.print("M: "); for $(i=0 ; i$ \&lt; Index $-1 ; i++)$ System.out.printf("\%3s ", a);

\} System.out.println("'");

During the calculation process of the serial, it needs to design cycle counting variable (K),

public static void ShellSort(int Index) \{

$$
\text { int } i, j, k \text {; }
$$$$
\text { int Temp; }
$$

Define change of the data, interval length of the divided cluster, and pointer of the process implementation:

Boolean Change; int DataLength;

int Pointer;

Initial cluster interval length and define whether the serial could be divided:

DataLength = (int) Index / 2;

while (DataLength $!=0)$

\{

Process each cluster, value of the temporarily stored Data[j], （a[j]） used at value exchange, calculate pointer to process:

$$
\begin{aligned}
& \text { for }(j=\text { DataLength; } j \text { \&lt; Index } ; j++)\{ \\
& \quad \text { Change }=\text { false; } \\
& \quad \text { Temp }=a[j] ; \\
& \quad \text { Pointer }=j-\text { DataLength; }
\end{aligned}
$$

Implement comparison and value exchange for the value within the cluster:

while (Temp \&lt; a[Pointer] \&\& Pointer >= 0 \&\&

Pointer \&lt; = Index $\{$ \{

$a[$ Pointer + DataLength $]=a[$ Pointer $]$;

Calculate the pointer of the next process:

Pointer = Pointer - DataLength;

Change = true;

if (Pointer \&lt; $0 \|$ Pointer > Index)

\} break;

Exchange with the last value:

$a[$ Pointer + DataLength $]=$ Temp;

if (Change) \{

After exporting current sorting result, calculate the interval length of next divide in the serial:

DataLength $=$ DataLength $/ 2$;

\} 


\section{Test Result Output}

The handling process in the business flow of the information data in the Unified Information System based on IOT is a complicated, unsorted, and undefined process, during the system construction process it's hard to implement big scale testing on the data source by practical information collection, instead it can only adopt virtual data input to replace manual data input. The hardware environment of the testing takes a high performance multiple cores server as hardware platform, adopts Vmware server version to create multiple systems to realize servers of each function, in the testing system it uses programming method to do data input of virtual system. Part of the codes in the testing programming is shown as following:

$$
\text { public static void main(String[] args) }
$$

\{

SortTool tool = new SortToolInteger(); Create testing data:

$$
\text { Integer }
$$

nData[]

$\{3,8,12,6,1,4,7,11,17,5,19,10,0,14,20,31,15,0,23,2\}$;

Integer $n$ Data[] =new Integer [500000];

for(int $i=0 ; i<500000 ; i++)\{$

$n$ Data $[i]=500000-i$;

\}

ShellSorter shellSorter $=$ new ShellSorter();

long time1 = System.currentTimeMillis();

Call Shell Sort algorithm, $Y$ represents time difference:

shellSorter.Sort(nData, tool ,false);

long sub = System.currentTimeMillis() - time1;

System.out.println("Y: "+sub+"ms");

Design for (int $\mathrm{j}=0 ; \mathrm{j}<5000 ; \mathrm{j}++$ ) $\{$; for (int $\mathrm{i}=0$; $\mathrm{i}<500$; $++\mathrm{i})$; System.out.printf("\%D ", nData[j*500+i]) etc, for sorted data group it adopts improved Shell Sort algorithm to perform system testing. The testing output is shown in table 1:

TABLE I. SYSTEM TESTING RESULTS

\begin{tabular}{llcc}
\hline Test & $\begin{array}{l}\mathbf{5 0 0 0 0 0} \\
\text { Elements }\end{array}$ & $\begin{array}{c}\mathbf{3 0 0 0 0 0} \\
\text { Elements }\end{array}$ & $\begin{array}{c}\mathbf{1 0 0 0 0 0} \\
\text { Elements }\end{array}$ \\
\hline $\begin{array}{l}\text { System prior to the } \\
\text { improvement } \\
(\mathrm{ms})\end{array}$ & 639613 & 392751 & 119293 \\
$\begin{array}{l}\text { Shell sorting } \\
\text { algorithm (ms) }\end{array}$ & 316 & 197 & 59 \\
\hline
\end{tabular}

In the testing, define 500000、300000、100000 initial testing elements respectively, in way of unsorted inverted sequence sorting data group to perform testing, implement sorting comparison testing on algorithms before system improvement and after adopting improved Shell Sort. It can be learnt from the table, there is big difference of sorting efficiency between systems before improvement and after adopting improved algorithm, the improved Shell Sort algorithm is very adaptive and feasible in the system.

\section{CONCLUSION}

The design and testing result of Logistics Unified Information System based on IOT indicates, in the IOT unified information process, the impact on time complexity of Shell Sort algorithm by increment serial is significantly bigger than those of different collecting devices as well as system data storage and other factors in the unified information, selecting proper increment serial and step length could significantly improve time efficiency of the sorting, adopting increment serial, jumpily moving inverted sequence record and other sorting methods could be beneficial to speed up process on the system data. Shell Sort algorithm applied to logistics system based on IOT technology, brings performance improvement on the Logistics Unified Information System, which represents the integrated development of information technology, and is the result of creative discussion on running mechanism of information system, it resolves the problems of the unsorted and sorted system operations of existing logistics supply chain information system etc, and points out the future development direction of IOT technology and intelligent logistics system.

\section{REFERENCES}

[1] X.Z LIN. Design and Implementation of Stowage Module of Unified Logistics Information System.IEEE, iTAP2010,2010,pp 1-5.

[2] ZHUWen-he.Realization ofWhole-process Intelligent Supply Chain Distribution ServicesBased on the Internet of Things[J].Logistics Technology.2010,(13):172-173.

[3] ZHANG Lian-tang,ZHANG Bo.Research of Best Increment Series on Shell's

[4] XZ LIN. Design and Realization of Concise Unified Logistics Information System[C]// Asia-Pacific Youth Conference On Communication Technology 2010. USA : Scientific Research Publishing.2010:414-418.

[5] LI Ming-hua, LI Chuan-zhong.An Economical and Practical Technology of Monitoring and Tracking Goods Based on GPS/GSM[J].Manufacture Information Engineering of China.2009,(21):44-47.

[6] XZ LIN.Analysis on Security Strategy of Double-factor Authentication in Unified Logistics Information System [C]// National Teaching Seminar On Cryptography And Information Security. USA : Scientific Research Publishing.2010:421-425.

[7] WANG Zu-zhu,SHEN Xiao-lu.Sorting Algorithms Based on C Language and Analysis[J].Journal of Anhui Electrical Engineer-ing Professional Technique College.2004,(1):85-89. 